\title{
Survey of the genus Phalangium Linnaeus, 1758 (Phalangiidae: Opiliones) from the Caucasus with description of two new species
}

\author{
Nataly Yu. SNEGOVAYA \\ Zoological Institute NAS of Azerbaijan, passage 1128, distr. 504. Baku. AZ 1073 Azerbaijan: \\ e-mail: snegovaya@yahoo.com
}

\begin{abstract}
Faunistic, distributional, and taxonomic data for the gemus Phalangium Linnaeus, 1758 from the Caucasus are presented. Eight species of the genus have been found in the region. Two new species: $P$. mcheidzeae from Georgia and $P$. gorbunovi from Volgograd region, Kalmikiya and Krasnodar region are described, the other six species of the area are $P$, opilio, $P$. punctipes, $P$. armatum, $P$. staregai. $P$. bakuense and $P$. armenicum. Each of them is illustrated, their main diagnostic features are summanzed and the occurrence of each species is presented. A key to the species of Phalangium of the region is provided.
\end{abstract}

Key words: harvestmen, Caucasus, Phalangium, morphological differences, identification key, Azerbaijan, Georgia

\section{INTRODUCTION}

The genus Phalangium was established by Linnaeus in 1758 and currently it consists of 21 valid species, i.e. P. opilio Linnaeus, 1758, P. savignyi Audouin, 1826, P. targionii (Canestrini, 1871), P. punctipes (L. Koch, 1878), P. clavipus Roewer, 1911, P. ligusticum (Roewer, 1923), P. licenti Schenkel, 1953 (?), P. wahrmanni Roewer, 1953, P. jakesi (Šilhavý, 1966), P. riedeli Starega, 1973, P. ghissaricum Gricenko, 1976, P. armatum Snegovaya, 2005, P. staregai Snegovaya. 2005, $P$. bakuense Snegovaya, 2006, $P$. vemustum Snegovaya, 2008, $P$. kopetdaghensis Tchemeris \& Snegovaya, 2012, P. armenicum Tchemeris, 2012, P. gromovi Tchemeris, 2012, P. kitabensis Tchemeris, 2012 and the two species newly found and described here $P$. mcheidzeae sp. n., $P$. gorbunovi $\mathrm{sp}$. n. Only the type-species Phalangium opilio Linnaeus, 1758 is widely distributed in nearly the whole Holarctic region and introduced to some areas in the Southern Hemisphere (Snegovaya, Starega, 2009). The known occurrences of other species is restricted to the Mediterranean Region, Central Asia and the Caucasus.

The species of the genus Phalangium from the Caucasus are dealt with by Koch (1878), Roewer (1911, 1912, 1923, 1956). Mcheidze (1959, 1962, 1964), Staręga (1966, 1978), Snegovaya (1999, 2004, 2005, 2006), Snegovaya \& Staręga (2011), Chemeris (2012). In total, six species of the genus Phalangium were known so far from the Caucasus - Phalangium opilio Linnacus, 1758, P. punctipes (L. Koch, 1878), P. armatum Snegovaya, 2005, P. staregai Snegovaya, 2005, P. bakuense Snegovaya, 2006, P. armenicum Chemeris, 2012.

While working on the collection of Opiliones from the Caucasian territory preserved in the National Museum of Georgia, Tbilisi (NMG) and the personal research collection of the author (RCNS), two new harvestmen species were recognized: $P$. mcheidzeae from Georgia and $P$, gorbunovi from Volgograd Region, Republic of Kalmykia and Krasnodar Region. They are described below. 
MATERIAL AND METHODS

Specimens were collected by manual method and pitfall traps and fixed in $75 \%$ ethanol, labeled, and transferred to the laboratory for further studies. Harvestmen were observed and measured using MBS-1 and Leica EZ4D stereomicroscopes and were photographed using a digital Sony DSC camera.

Abbreviations used: IZB (+ catalogue number) - Zoological Institute NAS of Azerbaijan, Baku; RCNS - Reference Collection of Nataly Snegovaya, Baku, Azerbaijan; NMG - National Museum of Georgia, Tbilisi, Georgia.

All measurements are in milimeters.

\title{
RESULTS
}

\section{Genus Phalangium Linnaeus, 1758}

The diagnosis of the genus Phalangium has been elaborated on by a number of authors (Roewer 1923: pp. 750-751, Šilhavý 1956: 218, Staręga 1976: pp. 367-368, Martens 1978: 231-232, Snegovaya \& Staręa 2009: 38). In this report, a slightly reworded diagnosis (Snegovaya \& Staręga, 2009) is followed.

Diagnosis. Eye mound relatively high (height $=$ length $=$ width), front and back surface of nearly equal width, deeply furrowed, with distinct denticles on eye rings. Body coloration yellowish with distinct brown/blackish saddle mark.

Chelicerae. $1^{\text {st }}$ segment without modifactions; $2^{\text {nd }}$ segment with dorsal apophysis, either thin or strong and very long, or sometimes lacking.

Pedipalps. Without apophysis (if any on patella, then very short), femur with weak armature mainly on dorsal side, in some species leg-like elongated and very thin.

Legs mostly long, femora with rows of denticles, $1^{\text {st }}$ leg thicker than the others.

Penis. Shaft thick, from the broad base gradually narrowing towards glans, with distinct subapical "spoon", glans triangular in profile.

Distribution. Mediterranean Region, Caucasus, Central Asia.

\section{Species survey}

\section{Phalangium opilio Linnaeus, 1758}

(Figs 1-16, 128)

\author{
Phalangium opilio: Mkheidze 1959: 113; \\ Phalangium opilio: Mkheidze 1964: 120; \\ Phalangium opilio: Staręga 1966: 401; \\ Phalangium opilio: Staręga 1978: 215; \\ Phalangium opilio: Chevrizov 1979: 22, ff. 115-118; \\ Phalangium opilio: Chemeris 1998: 198, ff. 49-58; \\ Phalangium opilio: Chemeris \& Kovblyuk 2005: 312.
}

Material: Russia. Adygea: $2 \hat{\partial}, 4 \uparrow q$, surroundings of Dakhovskaya, $465 \mathrm{~m}$ a.s.1., the Belaya river valley, $44.199^{\circ} \mathrm{N} 40.170^{\circ} \mathrm{E}, 29.06 .-01.07 .2009$, leg. K. Tomkovich (RCNS); 21 juv., Nikel, left bank of the Belaya river, 23.06.2010, leg. D. Volkova (RCNS). $1 \hat{\partial}$, NE of Dagonaki Plateau, "Kamennoe more" Mt. Ridge, ca. $1700 \mathrm{~m}$ a.s.1., under stones, 16.09.2003, leg. P. Kiyashko (RCNS); 1 ठ, 2 ๆq, 1 juv., Maykop Distr., 12 km S of Kamenomostskiy, Belaya river, edge of a beech wood, 22.08.2009, leg. A. Ponomarev (RCNS); $2 \delta \hat{\partial}, 10$ q, 3 juv., Caucasian Biospheric State reserve, 11-21 km SE of Guzeripl, Abago pasture, 1727-2010

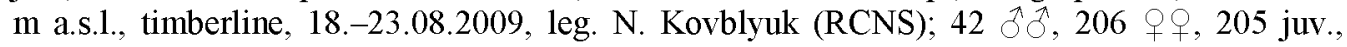
gramineous subalpine meadow, soil traps, March-October 2009, leg. Yu. Chumachenko 
(RCNS); Rostov Area: 2 + 9 Neklinovskiy Distr., Merzhavino, coast of Taganrog Bay, 01.07.2010, leg. A. Ponomarev (RCNS); 11 ¿ै coast of Taganrog Bay, 15.08.2006, leg. A. Ivliev (RCNS); 1 \&, Azov distr., coast of Taganrog Bay, Chumbur-Kosa, 02.07.2010, leg. A. Ponomarev (RCNS); 1 f Stephanidinodar, 02.07.2010, leg. A. Ponomarev (RCNS); Karachevo-Cherkessiya: $2 \hat{\jmath}, 4$ 9 , 2 juv. Teberda, 07.2010, leg. E. Khachikov (RCNS); Kabardino-Balkariya: $1 \hat{\delta}, 1$ \&, Subashi Mount, $3000 \mathrm{~m}$ a.s.1., 9.08.2006, leg. M. Nabozhenko (RCNS); North Ossetia: $2 \hat{\jmath}, 1+1.5 \mathrm{~km}$ E of Nuzal, ca. $1900 \mathrm{~m}$ a.s.1., pine forest, NorthEastern exposure, 24.08.2011, leg. Yu. Komarov (RCNS); Stavropol Area: $12 \hat{\delta} \bar{\delta}, 30$ q, 2 juv., surroundings of Izobilniy, 09.07.-08.08.2011, leg. V. Kozminikh (RCNS); $8 \hat{\jmath} \jmath, 21$ o 9 , same place and time (RCNS); Georgia: $4 \hat{\jmath} \hat{\jmath}, 2$ 우 Oni, 20.08.1975 (RCNS); $3 \hat{\jmath}, 3$ 우, 4 juv. Abkhazia, Tsebelda, 07.1914, leg. Y. Voronov (RCNS); $1 \hat{\delta}, 5$ 9 Khevsureti, $1500 \mathrm{~m}$ a.s.l., in the grass, 23.08.1987 (NMG N100-14, 863).

Description. See Y1git et al.(2007).

Distribution. Widespread in the Holarctic (native in Europe and Asia, introduced to USA, Canada and New Zealand) (Martens, 1978). In the Caucasus P.opilio occurs in North Caucasus and Georgia (Staręga 1978).

\title{
Phalangium punctipes (L. Koch, 1878)
}

(Figs 17-33, 128)

\author{
Zacheus cancasicus Mcheidze 1959: 113; \\ Zacheus cancasicus: Mcheidze 1962: 185; \\ Zacheus caucasicus: Mcheidze 1964: 121; \\ Phalangium punctipes: Staręga 1966: 401-402; \\ Phalangium punctipes: Staręga 1973: 134-135, ff. $13-15$; \\ Phalangium punctipes: Staręga 1978: 216-217; \\ Phalangium punctipes: Chevrizov 1979: 19, fig. 110-111; \\ Phalangium punctipes: Snegovaya 1999: 455-456, ff. 24-33; \\ Zachaeus crista: Snegovaya 1999: 458 (only IZB 63); \\ Phalangium punctipes: Snegovaya 2004: 316; \\ Phalangium punctipes: Chemeris \& Kovblyuk 2005: 307, 309-312, ff. 9-18; \\ Phalangium punctipes: Snegovaya $2010: 497-501$, ff. 1-7; \\ Phalangium punctipes: Snegovaya \& Starega 2011: 50.
}

Material: Azerbaijan, Absheron: $1 \delta, 1$ Shagan, under stones, 16.03.1994, leg. E. Guseinov (IZB 15); $1 \hat{\jmath}, 1$ Gara-Chukhur, 14. -23.06.1994, leg. N. Snegovaya (IZB 14); 5 $\delta$ Bailov, under stones, 29.04.1996, leg. N. Snegovaya (IZB 40); $2 \delta \hat{\delta}, 10$ juv. Baku, near Zoological Institute, 07.04.2003, leg. N. Snegovaya (IZB 138); 1 ઈ, 1 †, 3 juv. same place, 05.-09.05.2004, leg. Kh. Aliev (IZB 225); $76 \delta \hat{\delta}, 77 q 9,1$ juv. Yasamal valley, pitfall traps, April-October 2010, leg. N. Snegovaya (RCNS); $7 \hat{\delta}, 8$ qq environs of Baku, Volch'i

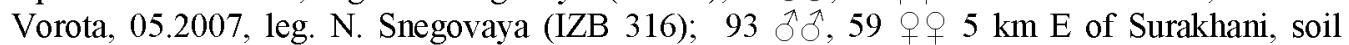
traps, April-October 2010, leg. N. Snegovaya (RCNS); Gobustan: 4 dै, 4 $q$ along BakuTbilisi-Jeyhan (BTC) oil pipeline, 17.-21.04.2003, leg. I. Alekperov (IZB 134); 1 ठิ, 2 qq (IZB 270, 272), Boyukdash, 11.-16.04.2005, leg. E. Guseinov; $1 \hat{\delta}$ (IZB 279), ibidem, 15.04.2005, leg. E. Guseinov; 1 d, 3 †q (RCNS), Jeyrankechmez, 11.05.2012, leg. D. Kasatkin; Gusar Distr.: 3 ठै (IZB 84), near Shakhdag Mt., $3000 \mathrm{~m}$ a.s.l., under stones, 06.08.2001, leg. N. Snegovaya, Kh. Aliev, E. Guseinov; 2 qq (IZB 86), Laza vill., 06.08.2001, leg. N. Snegovaya; Lerik Distr.: $1 \hat{\jmath}$ (IZB 119), Divagach, $1400 \mathrm{~m}$ a.s.l., 25.05.2003, leg. N. Snegovaya; $1 \hat{\jmath}$ (IZB 198), ibidem, leg. Yu. Marusik; $7 \hat{\jmath}, 6$ 6 (RCNS), environs of Gosmolyan vill., 18-20.05.2012, leg. D. Kasatkin; $1 \hat{\jmath}$ (RCNS), Peshtatyuk Vill., 22-23.05.2012, leg. D. Kasatkin; Lenkoran Distr.: 2 우 (IZB 327), Moscow forest, 21.05.2007, leg. N. Snegovaya; Astara Distr.: $3 \hat{\jmath} \hat{\jmath}, 2$ q⿻ (RCNS), Sim vill., 15-16.05.2012, 
leg. D. Kasatkun; Yardimli Distr:: $4 \hat{\delta} \hat{\delta}, 3$ $9+$ (IZB 365), environs of Kyurekchi vill., 26.05.2008, leg. N. Snegovaya; 1 $\delta$, $1 \uparrow$ (IZB 380), ibidem, leg. N. Snegovaya; $1 \succsim$ (IZB 381), ibidem, leg. N. Snegovaya; $1 \delta$ (IZB 368), Uzyubashi Mt. environs, 29.05.2008, leg. N. Snegovaya; Nakhchivan Autonomous Republic: $2 \delta \delta, 2$ 우, 3 juv. (IZB 181), May-June 2003, leg. Kh. Aliev; Shemakha Distr.: 3 우 (IZB 392), Soyugbulag, 16.06.2008, leg. N. Snegovaya; 2 우 (GNM A3-09), Shemakha town environs, 14.07.1973, leg. E. Didmanidze; 2 $\hat{\delta}$ (GNM, N25-07, 843, 681), Pirsagat Valley, 05.04.1902, leg. Shmidt, Shelkovnikov; 5 đo d, 2 우우 (N25-07, 886), ibidem, 06.04.1907; $1 \hat{\delta}$ (GNM N 22-08, 934), Sulut-chay river gorge, 07.05.1908, leg. Shelkovnikov, Voronov; Agdash Distr.: $4 \hat{\jmath} \hat{\jmath}, 2$ 우 (GNM A3 -06), Turianchay State reserve, 08.07.1973, leg. R. Tartanishvili; Khachmaz Distr.: $1 \AA$ (GNM A302), environs of Khachmaz town, 18.07.1973, leg. R. Tartanishvili; $2 \hat{\jmath}$ (GNM N25-07, 869), N Mughan, Shmidt, leg. Shelkovnikov; Russia, Rostov Area: $1 \hat{\jmath}$ (RCNS), Ust'-Donetskiy Distr.: Razdorskaya vill., steppe, 24.04.2002, leg. A. Ponomarev; $3 \hat{\jmath}, 4$ 9 , 6 juv. (RCNS), ibidem, 05.05-26.07.2003, leg. A. Ponomarev; $20 \hat{\jmath} \hat{\jmath}, 13$ +9 (RCNS), ibidem, 3.052.06.2003, leg. A. Ponomarev; $14 \hat{\jmath} \hat{\jmath}, 43$ + ,, 8 juv. (RCNS), Orlovskiy Distr.: environs of Manich settlement, 14-21.05.2009, leg. A. Ponomarev, V. Perepechenkov; Georgia: $1 \hat{\jmath}, 2$ q Manglisi, 06.07.1965 (NMG); 4 우 Kobuleti, 1956 (NMG N1 144); 1 q Mutso, Khevsureti, river bank, 26.08.1988, leg. M. Intskirveli (NMG); $1 \curvearrowright, 2$ 을 2 repash'e ozero near Tbilisi, 03.04.1913, leg. Zaitsev (NMG N13, 922); 2 ठ̊, 1 i ibidem, 19.04.1908, leg. Satunin (NMG N 18-08); $1 \hat{\partial}$ environs of Tbilisi, March 1907, leg. Satunin (NMG N 18-08, 938); $1 \hat{\partial}, 1$ + 4 juv. environs of Tbilisi, Mukhrovani, 26.03.1950 (NMG N1138); $1 \hat{\jmath}$, Svanetiya, July 1911, leg. Shelkovnikov (NMG N55-11, 932); 1 d, 1 environs of Telavi, April 1907, leg. Fursov (NMG N9-07, 861); 1 ठ Kiketi, 13.07.1989, leg. M. Ukraintsev (NMG 1556).

Description: See Chemeris \& Kovblyuk (2005) and Snegovaya (2010).

Distribution. Widespread throughout all of the Caucasus.

\section{Phalangium armatum Snegovaya, 2005}

(Figs 34-50, 128)

Phalangium savignyi: Snegovaya 1999: 455;

Phalangium savignyi: Snegovaya 2004: 313;

Phalangium armatum: Snegovaya 2005: 22-26, fig. 17-34;

Phalangium zuvandicum: Snegovaya 2005: 23, 26, fig. 35-45;

Phalangium armatum: Snegovaya \& Staręga 2011: 50

Material: Azerbaijan, Lerik Distr.: 2 ㅇ (IZB 53), Zuvand, under stones, 14.06.2006, leg. N. Snegovaya, O. Gorbunov; $1 \hat{\jmath}, 1$ juv. (IZB 120), $5 \mathrm{~km}$ from Divagach vill., near river, 26.05.2003, leg. N. Snegovaya; $4 \hat{\delta} \hat{\jmath}, 10+9$, 1 juv. (IZB 124), Pirasora vill., 1740-1900 m a.s.1., 25.05.2003, leg. N. Snegovaya; 4 9 (IZB 189), Zuvand area, ca. $3 \mathrm{~km} \mathrm{~W}$ of Lerik town, 38 $43^{\prime} 834^{\prime \prime} \mathrm{N} 48^{\circ} 25^{\prime} 669^{\prime \prime} \mathrm{E}, 1200 \mathrm{~m}$ a.s.1., slopy meadows, 25.05.2003, leg. Yu. Marusik; $2 \hat{\delta}$ (RCNS), $6 \mathrm{~km}$ N of Gosmalyan vill., 09.05.2004, leg. N. Snegovaya; $1 \hat{\text { (IZB }}$ 297), Gosmalyan vill., 28.05.2005, leg. N. Snegovaya; 4 웅 (IZB 337), ibidem, 12.06.2007, leg. N. Snegovaya; 1 (IZB 339), ibidem, 11.06.2007, leg. N. Snegovaya; 3 o (IZB 347), ibidem, 12.06.2009, leg. N. Snegovaya; $4 \hat{\jmath} \hat{\jmath}, 4$ 우, 1 juv. (RCNS), ibidem, 18-20.05.2012, leg. D. Kasatkin; 3 q 0 (RCNS), near Devil bridge, 14.06.2007, leg. N. Snegovaya; $6 \hat{\jmath} \hat{\delta}, 4$ 우우 (RCNS), ibidem, 21.05.2012, leg. D. Kasatkin; Yardimli Distr.: 1 (IZB 357), environs of Kyurekchi settlement, ca. $1500 \mathrm{~m}$ a.s.1., 25.05.2008, leg. N. Snegovaya; $9 \delta \hat{\delta}, 2$ 우 (IZB 361-362), ibidem, 26.05.2008, leg. N. Snegovaya, D. Kasatkin; 1 ठ, 1 (IZB 372), near Uzyubashi Mt., 29.05.2008, leg. N. Snegovaya, D. Kasatkin; $2 \hat{\jmath} \hat{\partial}, 1$ q (IZB 376), ibidem, 26-

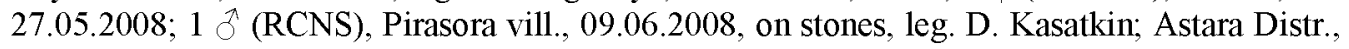


1 (RCNS), Sim vill., 15-16.05.2012, leg. D. Kasatkin; Nakhchivan Autonomous Republic: 2 İ (RCNS), Bichenek, Shakhbuz National Park, 23.06.2012, leg. N. Snegovaya.

Description: See Snegovaya (2005).

Distribution. The species is recorded from Lerik, Yardimli, Astara, and Nakhichevan (Azerbaijan).

\section{Phalangium staregai Snegovaya, 2005}

(Figs 51-66, 129)

Phalangium staregai Snegovaya 2005: 26-29, f, 46-62;

Phalangium staregai: Snegovaya \& Staręga 2011: 50;

Material: Azerbaijan, Lerik Distr: 1 \& 5 q , 1 juv. (IZB 136), $1200 \mathrm{~m}$ a.s.1., 25.05.2003, leg. N. Snegovaya; 1 (RCNS). Zuvand, environs of Pirasora village., 1700-2000 m a.s.1., 25-26.05.2003, leg. Yu. Marusik; 1 đ. 7 † (IZB 331), Gosmalyan vill., 11.06.2007, leg. N. Snegovaya, 18, 3 q (IZB 330), ibidem, 12.06.2007, leg. N. Snegovaya; 5 q (IZB 389), Pirasora vill., 07. -09.06.2008, leg. D. Kasatkin; 1 . 3.90 (RCNS), Yukhari Amburdarya vill., 08.05.2004, leg. N. Snegovaya; Yardimli Distr.: 3 \%. 3 q (IZB 382), environs. of Uzyubashi Mt., 29.05.2008, leg. N. Snegovaya; 1 (NMG N26-07, 873), Shirin-Kum, Garabakh steppe, 18.04.1906, leg. Kaznakov, Shelkovnikov; 4 3े. 3 क क (NMG N26-07, 810), Peygambar, Garabakh steppe, 19.04.1907, leg. Kaznakov \& Shelkovnikov; 9 \& \&, 12 + (NMG N26-07, 876), Mil steppe. 17.04.1907. leg. Kaznakov\& Shelkovnikov.

Description: See Snegovaya (2005).

Distribution. The species is currently only known from Azerbaijan.

\section{Phalangium bakuense Snegovaya, 2006}

(Figs 67-88, 129)

\section{Phalangium bakiensis: Snegovaya 2006; 95-99, f, 1-16}

Material: Azerbaijan, Absheron: $2 \nexists \delta, 1 q$ (IZB 305), environs of Baku city, Volch"i Vorota, 10.05.2006, leg. N. Snegovaya; 3 d ठ (IZB 318), ibidem, 05.2006, leg. N. Snegovaya; 10 (I) (IZB 313), Vishnevka, 09.05.2006, leg. N. Snegovaya; 1 d, 1 q (IZB 317), ibidem, 12.05.2007, leg. N. Snegovaya; $4 \delta \delta, 3 \phi, 1$ juv. (RCNS), Yasamal valley, April-October 2010, pitfall traps, leg. N. Snegovaya; $41 \delta \delta, 42$ q. 51 juv. (RCNS), near Surakhani settlement, April-October 2010, pitfall traps. leg. N. Snegovaya; $3 \delta \delta, 3 \rho f$ (IZB 278), Gres "Severnaya", 01.05.2005, leg. E. Guseinov.

Description: See Snegovaya (2006).

Distribution. This species was described from Absheron Peninsula (Azerbaijan). It has not been recorded from any other locality.

\section{Phalangium armenicum Chemeris, 2012}

(Figs 84-98, 129)

Phalangium armenicus: Chemeris 2012: 13-17, Figs. 1-2, 9-10, 17-19, 22-23, 28

Material $2 \partial \theta, 2 q q$ (RCNS), Azerbaijan, Nakhchivan Autonomous Republic, Julfa, Dizya, on the wall, 26.04.2012, leg. E. Guseinov.

Description: See Chemeris (2012).

Distribution. The species was originally found and described from Armenia. Herein it is further recorded from Nakhichevan AR (Azerbaijan). 


\section{Phalangium mcheidzeae sp. $\mathbf{n}$.}

(Figs 99-112, 129)

Material: Georgia, 1 holotype (NMG N994), Kiketi, 02.07.1963, leg. T. Mcheidze; 1 , 1 paratypes (NMG N994), ibidem.

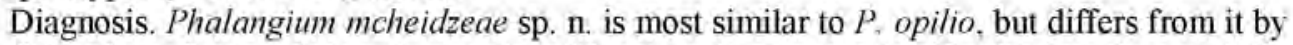
the following characteristics: legs only with setae, leg I considerably thickened, especially femur (Figs. 99-100, 112): legs brown; femora of all legs cylindrical, except femur I pentagonal in cross section; male cheliceral apophysis rigid; penis shaft with equally spaced lateral edges, unlike $P$. opilio with laterally curved shape of the penis; body covered only by setae and small denticles (Figs. 99, 109-111).

Male body length 6.4, width 4.0. Body quadrangular to elongated oval in shape, surface covered with small denticles (Figs. 99-100). Saddle shape mark, brownish. Anterior part of cephalothorax brown. Eye mound relatively small, rounded, with 6 denticles on each side. light-brown. Area infront of eye mound with few large denticles. Chelicera relatively large. with medium sized, distally attemuated, rigid apophysis (Figs. 102-103). Basal segment of chelicera dorsally with a few denticles and setae, distal segment dorsally with differently sized denticles and setae. Lengths of basal segment 2.5, distal segment 6.0, and aphophysis 3.0. Pedipalp long, femur dorsally with small hair-tipped denticles, other segments with setae only (Fig. 106). Pedipalp segment lengths: $6.6+3.2+2.0+6.7=18.5$ total.

Legs relatively long, leg I, especially femur I, considerably thickened. Legs brown. Femur I dorsally with small denticles, other femora only with setae. Tibia and patella ventrally with large denticles, other leg articles with very small setae. Lengths of legs $\mathrm{I}-5.4+2.0+4.5+$ $5.0+7.0=23.9 ;$ II $-7.6+1.7+6.6+7.7+12.3=35.9 ;$ III $-5.1+1.6+4.0+6.0+8.0=$ $24.7 ;$ IV $-7.2+2.0+5.2+9.5+11.6=35.5$.

Penis. Corpus long, wings relatively narrow, glans relatively narrow (Figs. 109-111). Penis length 3.3 , glans 0.35 , stylus 0.13 .

Female differs from male by having more numerous large denticles in front of eye mound, absence of armament (denticles on femur to metatarsus) on legs, thinner leg femora. absence of cheliceral apophysis and much shorter pedipalps (Figs. 101, 104-105, 107-108). Female body length 6.6 , width 4.0 . Lengths of legs: $\mathrm{I}-3.7+1.3+3.5+4.2+6.4=19.1$; II $-7.5+1.2+6.0+$ $6.2+13.2=34.1 ; \mathrm{III}-4.6+1.4+3.5+5.1+7.3=21.9 ; \mathrm{IV}-7.6+1.5+5.0+8.8+11.0=33.9$.

Distribution. The species is known only from Georgia.

Etymology. The species was named in honor of the deceased Georgian arachnologist. Dr. Tamara Mcheidze (Marusik \& Otto 2008).

\section{Phalangium gorbunovi sp. $\mathrm{n}$.}

(Figs 113-127. 129)

Material. 1 holotype, 19 paratype (ZMMGU), $3 \AA \AA, 1 \rho$ (paratypes - RCNS), Russia,

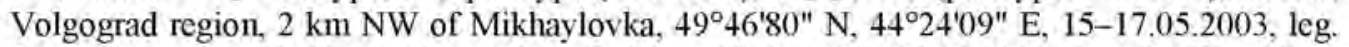
O. Gorbunov; 1 \&, Krasnodar region, Bolshoy Utrish, 01.-05.05.2008, leg. E. Khachikov (RNCS); 1 . Kalmikiya, environs of Ulan-Khol, 12.05.2007, leg. E. Terskov (RCNS).

Diagnosis. This new species differs from the closest related species Phalangium punctipes by much thinner pedipalps, less thickened femur $I$, the presence of longitudinal rows of large denticles on the legs, especially on femur I (Fig. 127), and differently shaped penis with wider apical part, "spoon", in dorsal view (Figs 124-126).

Description. Body length 5.2. width 3.0. Large harvestmen, with quadrangular shaped body (Figs 113-114). Tergites with large black-tipped denticles. In front of eye mound a group of similar denticles. Eye mound round, high, with 9 large denticles on each side. Body light 
brownish with pronounced saddle-shaped mark. Legs not very long, with longitudinal rows of large denticles. All femora cylindrical, femur I thickened (Fig. 127). Lengths of legs: I $-4.5+$ $1.6+4.0+5.0+7.4=22.5 ; \mathrm{II}-7.0+1.6+6.2+6.6+11.6=33.0 ; \mathrm{III}-4.5+1.6+4.0+5.2$ $+8.2=23.5 ; \mathrm{IV}-6.2+1.6+4.0+4.6+15.0=31.4$. Pedipalp long, all segments covered with setae (Figs. 120-121). Pedipalp lengths $4.2+1.5+2.0+4.7=12.4$. Chelicera large, both segments anteriory covered with denticles (Figs. 116-117). Lengths of basal segment 2.8, distal segment 3.1. Penis typical for Phalangium with wide apical part and narrow wings (Figs. 124126). Penis length 3.1 , glans 0.3 , stylus 0.15 .

Female differs from male by larger, more rounded body, shorter pedipalps and smaller chelicerae (Figs. 115, 118-119, 122-123). Female body length 7.6 , width 3.6 . Pedipalp length $1.6+0.8+1.0+2.1=5.5$. Basal cheliceral segment length 1.8 , distal segment 2.1. Lengths of legs: $\mathrm{I}-3.2+1.5+3.0+3.5+6.2=17.4 ; \mathrm{II}-5.0+1.5+4.6+5.0+10.5=26.6 ; \mathrm{III}-4.0+$ $1.5+3.2+4.0+7.0=19.7 \div$ IV $-5.5+1.5+4.1+7.0+10.4=28.5$.

Distribution. The species has been found only in the Volgograd province, Kalmikiya, Krasnodar area (Russia).

Etymology. The species was named in honor of the famous Russian entomologists Dr. O.

G. Gorbunov (Moscow. Russia), who collected most of the known specimens of this species.

COMPARATIVE MORPHOLOGICAL ANALYSIS

The species of the genus Phalangium from the Caucasus differ by following featuers:

\begin{tabular}{|c|c|c|c|c|}
\hline Species & Body & Ocularium & Chelicera & Podipalps \\
\hline P. opilio & $\begin{array}{l}\text { Quadrangular, } \\
\text { covered with short } \\
\text { black-tipped tubercles }\end{array}$ & $\begin{array}{l}\text { Rounded, with two } \\
\text { rows of large } 6-8 \\
\text { black-tipped } \\
\text { tubercles on each side }\end{array}$ & $\begin{array}{l}\text { Large, with different size } \\
\text { cheliceral hom, wide and } \\
\text { robust }\end{array}$ & $\begin{array}{l}\text { Long, covered with hairs: } \\
\text { patella with a small } \\
\text { apophysis }\end{array}$ \\
\hline P.punctipes & $\begin{array}{l}\text { Quadrangle, covered } \\
\text { with short hairs and } \\
\text { small black-tipped } \\
\text { tubercles }\end{array}$ & $\begin{array}{l}\text { Round, carries two } \\
\text { rows of } 8-12 \\
\text { tubercles on each side }\end{array}$ & $\begin{array}{l}\text { Strong, segment II in male } \\
\text { without a process, covered } \\
\text { with tubercles and hairs }\end{array}$ & $\begin{array}{l}\text { Moderate length, femora } \\
\text { with 3-5 basal tubercles }\end{array}$ \\
\hline P.armatum & $\begin{array}{l}\text { Short, rounded- } \\
\text { quadrangular, covered } \\
\text { with tall, black-tipped } \\
\text { tubercles }\end{array}$ & $\begin{array}{l}\text { Crown-shaped, } \\
\text { dorsally with two } \\
\text { rows of } 10-11 \text { tall } \\
\text { tubercles on each side }\end{array}$ & $\begin{array}{l}\text { Large with different size } \\
\text { (usuallu very long) } \\
\text { cheliceral hom, narrow }\end{array}$ & $\begin{array}{l}\text { Long covered with } \\
\text { longitudinal rows of } \\
\text { tubercles, tarsi covered with } \\
\text { hairs, apophyses not distinct }\end{array}$ \\
\hline P.staregai & $\begin{array}{l}\text { Quadrangle, widening } \\
\text { fowards the caudal end. } \\
\text { covered with hairs and } \\
\text { small denticles }\end{array}$ & $\begin{array}{l}\text { Rounded, with } 10-11 \\
\text { small setae on each } \\
\text { side }\end{array}$ & $\begin{array}{l}\text { Segment II in male with } \\
\text { long, bent and whip- } \\
\text { shaped process }\end{array}$ & $\begin{array}{l}\text { Moderate length, covered } \\
\text { with hairs and micro- } \\
\text { denticles }\end{array}$ \\
\hline P. bakuense & $\begin{array}{l}\text { Quadrangular, oval, } \\
\text { not very large, } \\
\text { covered with hairs } \\
\text { and small denticles }\end{array}$ & $\begin{array}{l}\text { Rounded with } 2-3 \\
\text { rows of black-tippod } \\
\text { tubercles }\end{array}$ & $\begin{array}{l}\text { Relatively small, covered } \\
\text { with setae and small black- } \\
\text { tipped denticles and } \\
\text { microdenticles }\end{array}$ & $\begin{array}{l}\text { Not very long covered with } \\
\text { small denticles and setae, } \\
\text { patella bears hardly visible } \\
\text { apophysis }\end{array}$ \\
\hline$P$-armenicum & $\begin{array}{l}\text { Medium size, coverad } \\
\text { with black-tipped } \\
\text { denticles }\end{array}$ & $\begin{array}{l}\text { Rounded, wifh 6-9 } \\
\text { tubercles on each side }\end{array}$ & $\begin{array}{l}\text { Distal segnent of chelicera } \\
\text { elongated, without } \\
\text { apophysis, densely covered } \\
\text { with numerous black- } \\
\text { tipped denticles }\end{array}$ & $\begin{array}{l}\text { Short, femur dorsally with } \\
\text { scattered black-tipped } \\
\text { denticles, tibia ventrally on } \\
\text { the apical part armed of } 2-3 \\
\text { tiny denticles }\end{array}$ \\
\hline P. mcheidzeae & $\begin{array}{l}\text { Quadrangular, } \\
\text { covered with small } \\
\text { denticles }\end{array}$ & $\begin{array}{l}\text { Not very large, } \\
\text { rounded, canaliculate, } \\
\text { with } 6 \text { small denticles } \\
\text { on each side }\end{array}$ & $\begin{array}{l}\text { Relatively large, with } \\
\text { middle sizod, attenuated but } \\
\text { rigid, apophyses, coverod } \\
\text { with denticles and setae }\end{array}$ & $\begin{array}{l}\text { Long femora dorsally with } \\
\text { small hair-fipped denticles } \\
\text { other segments with setae } \\
\text { only }\end{array}$ \\
\hline P. gorbunovi & $\begin{array}{l}\text { Large, quadrangular, } \\
\text { covered with large } \\
\text { black-tippod dentilces }\end{array}$ & $\begin{array}{l}\text { Round, high, with } 9 \\
\text { large denticles on } \\
\text { each side }\end{array}$ & $\begin{array}{l}\text { Large, both segments } \\
\text { dorsally covered with } \\
\text { denticles }\end{array}$ & $\begin{array}{l}\text { Long all segments covered } \\
\text { with setae }\end{array}$ \\
\hline
\end{tabular}




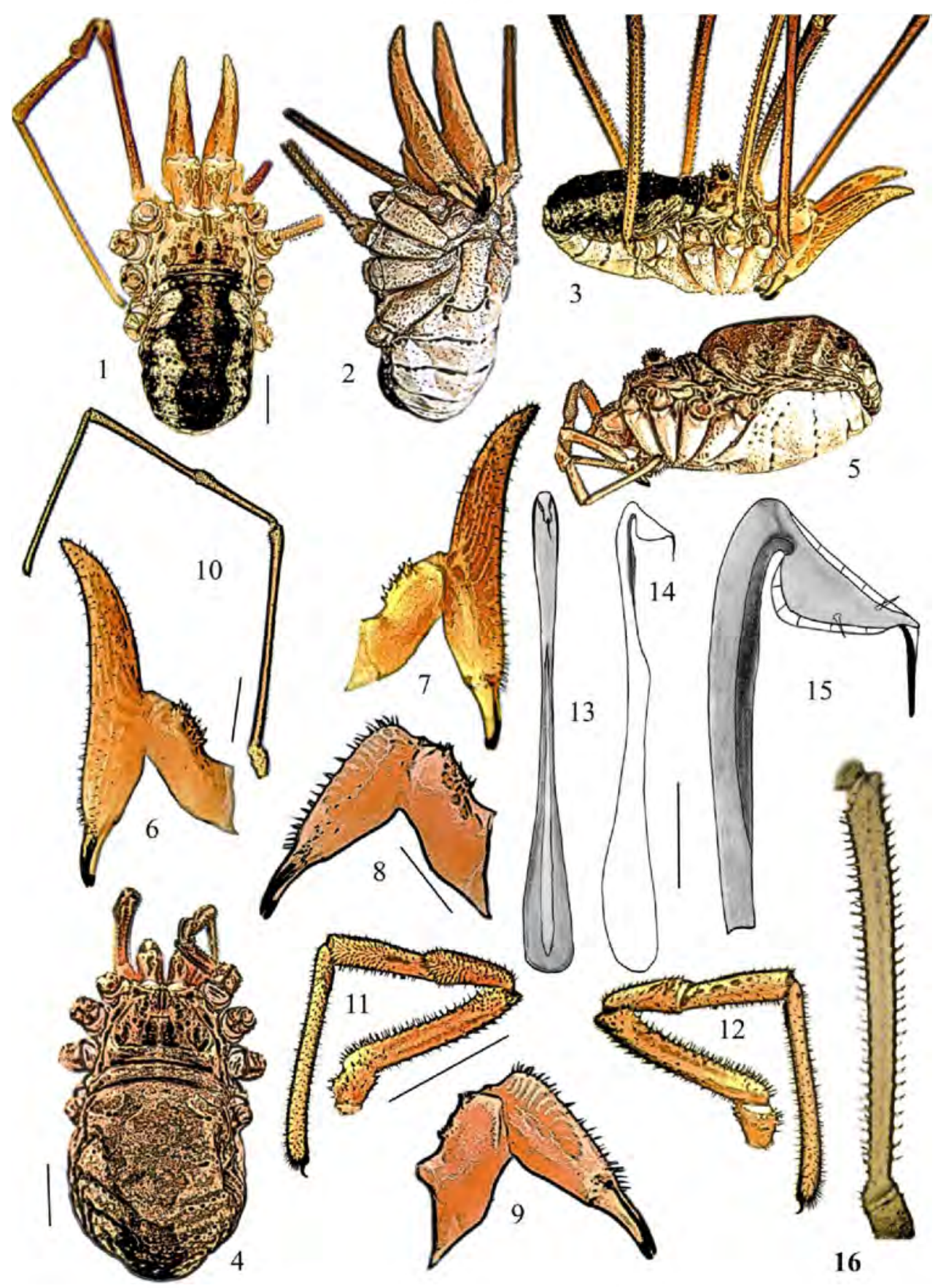

Figs 1-16. Phalangium opilio Linnaeus, 1758: 1 - male body, dorsal view, 2 - male body, anterolateral view of the ventral side, 3 - male body, lateral view, 4 - female body, dorsal view, 5 - female body, lateral view, 6 - male chelicera, lateral view, 7 - male chelicera, lateral view, 8 - female chelicera, lateral view, 9 - female chelicera, lateral view, 10 - male pedipalp, lateral view, 11 - female pedipalp, lateral view, 12 - female pedipalp, lateral view, 13 penis, dorsal view, 14 - penis, lateral view, 15 - glans, lateral view, 16 - femur I. Scale bars $=1 \mathrm{~mm}$. 
Phalangium of Caucasus

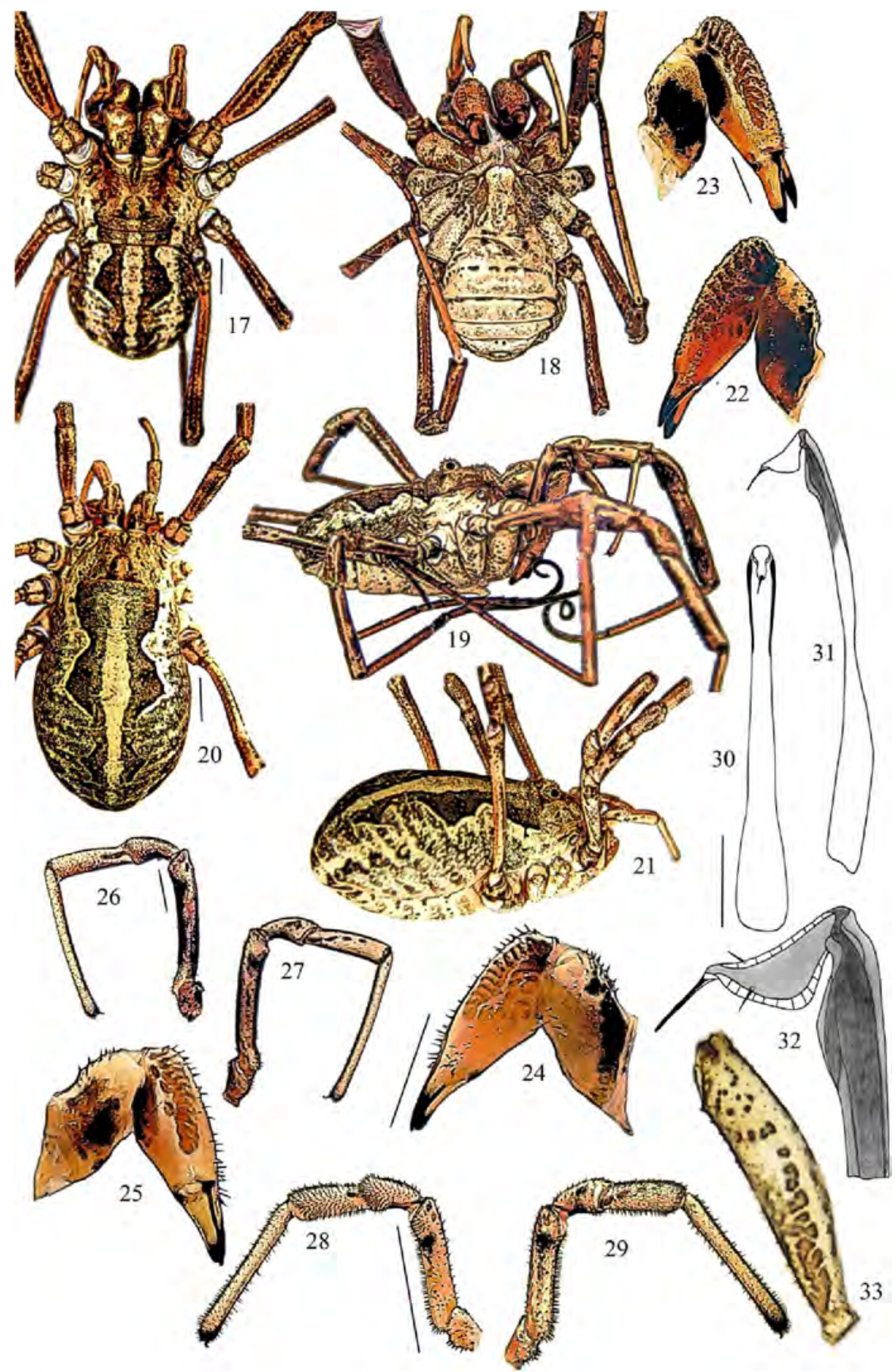

Figs 17-33. Phalangium punctipes (L. Koch, 1878): 17 - male body, dorsal view, 18 - male body, ventral view, 19 male body, lateral view, 20 - female body, dorsal view, 21 - female body, dorsolateral view, 22 - male chelicerae, lateral view, 23 - male chelicerae, lateral view, 24 - female chelicerae, lateral view, 25 - female chelicerae, lateral view, 26 male pedipalp, lateral view, 27 - male pedipalp, lateral view, 28 - female pedipalp, lateral view, 29 - female pedipalp, lateral view, 30 - penis, dorsal view, 31 - penis, lateral view, 32 - glans, lateral view, $33-$ femur I. Scale bars $=1 \mathrm{~mm}$. 


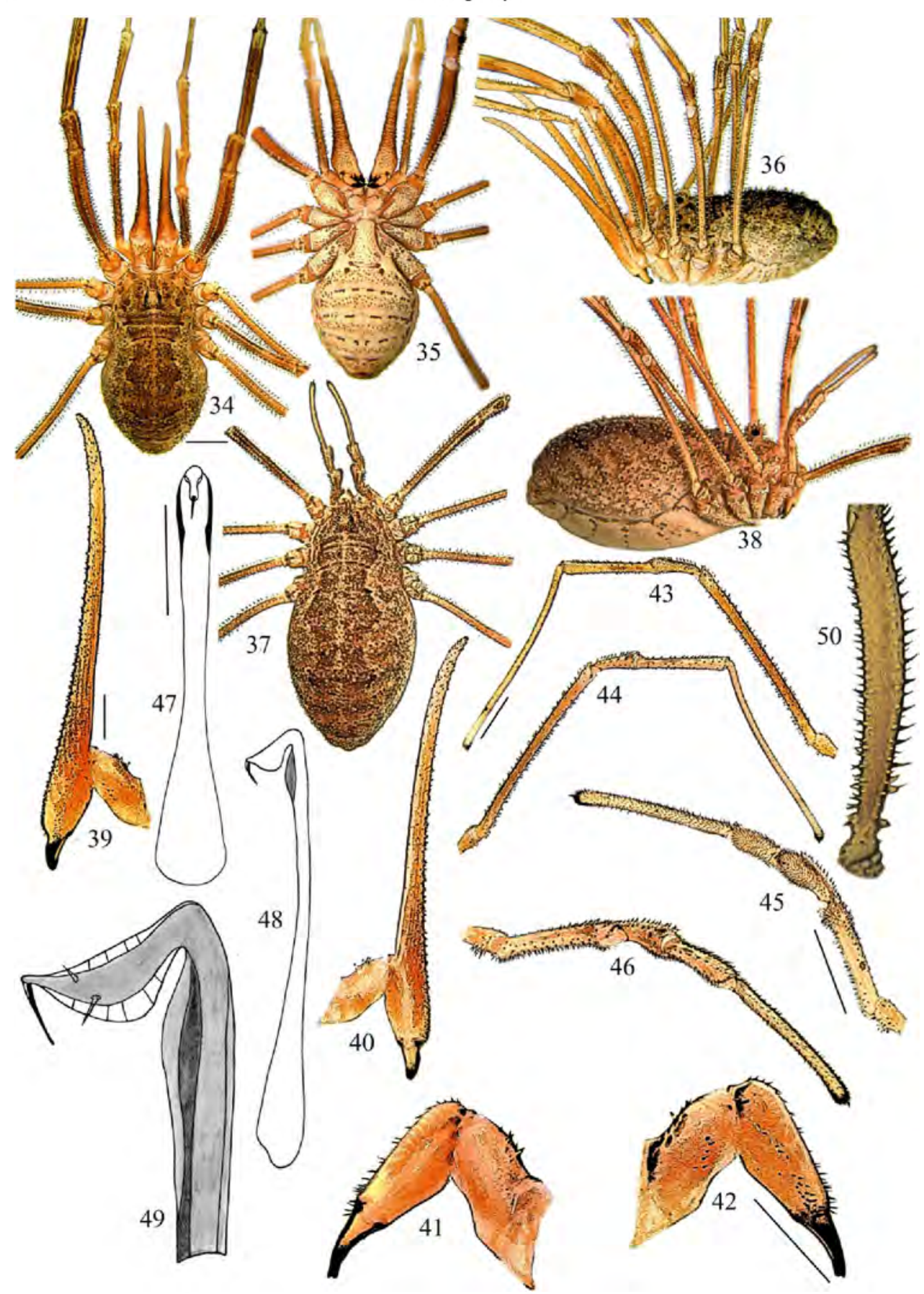

Figs 34-50. Phalangium armatum Snegovaya, 2005: 34 - male body, dorsal view, 35 - male body, ventral view, 36 male body, lateral view, 37 - female body, dorsal view, 38 - female body, lateral view, 39 - male chelicerae, lateral view, 40 - male chelicerae, lateral view, 41 - female chelicerae, lateral view, 42 - female chelicerae, lateral view, 43 male pedipalp, lateral view, 44 - male pedipalp, lateral view, 45 - female pedipalp, lateral view, 46 - female pedipalp, lateral view, 47 - penis, dorsal view, 48 - penis, lateral view, 49 - glans, lateral view, $50-$ femur I. Scale bars $=1 \mathrm{~mm}$. 


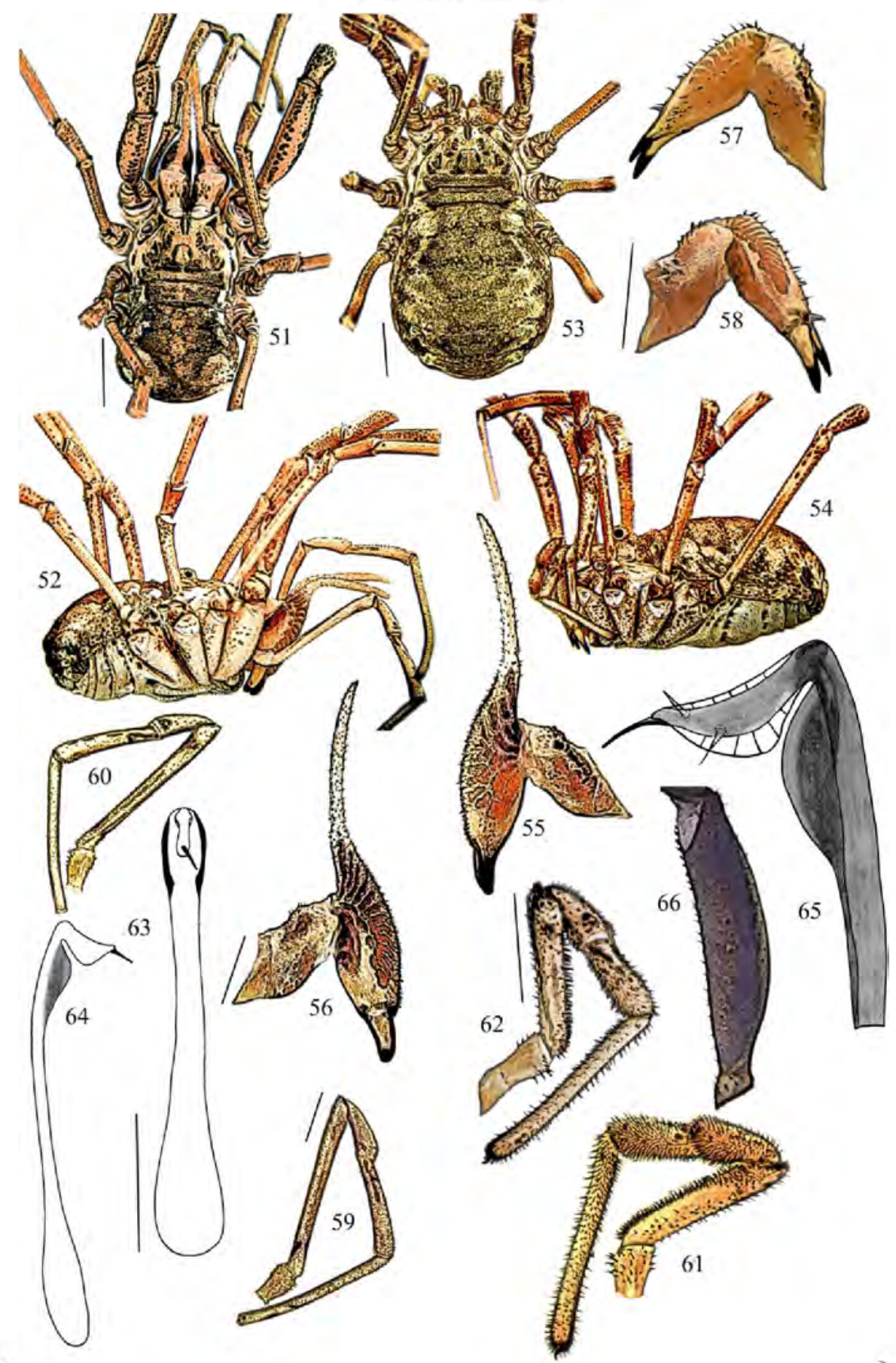

Figs 51-66. Phalangium staregai Snegovaya, 2005: 51 - male body, dorsal view, 52 - male body, lateral view, 53 female body, dorsal view, 54 - female body, lateral view, 55 - male chelicerac, lateral view, 56 - male chelicerae, lateral view, 57 - female chelicerae, lateral view, 58 - female chelicerae, lateral view, 59 -male pedipalp, lateral view, 60 -male pedipalp, lateral view, 61 - female pedipalp, lateral view, 62 - female pedipalp, lateral view, 63 - penis, dorsal view, 64 - penis, lateral view, 65 -glans, lateral view, 66 - femur I. Scale bars - $1 \mathrm{~mm}$. 

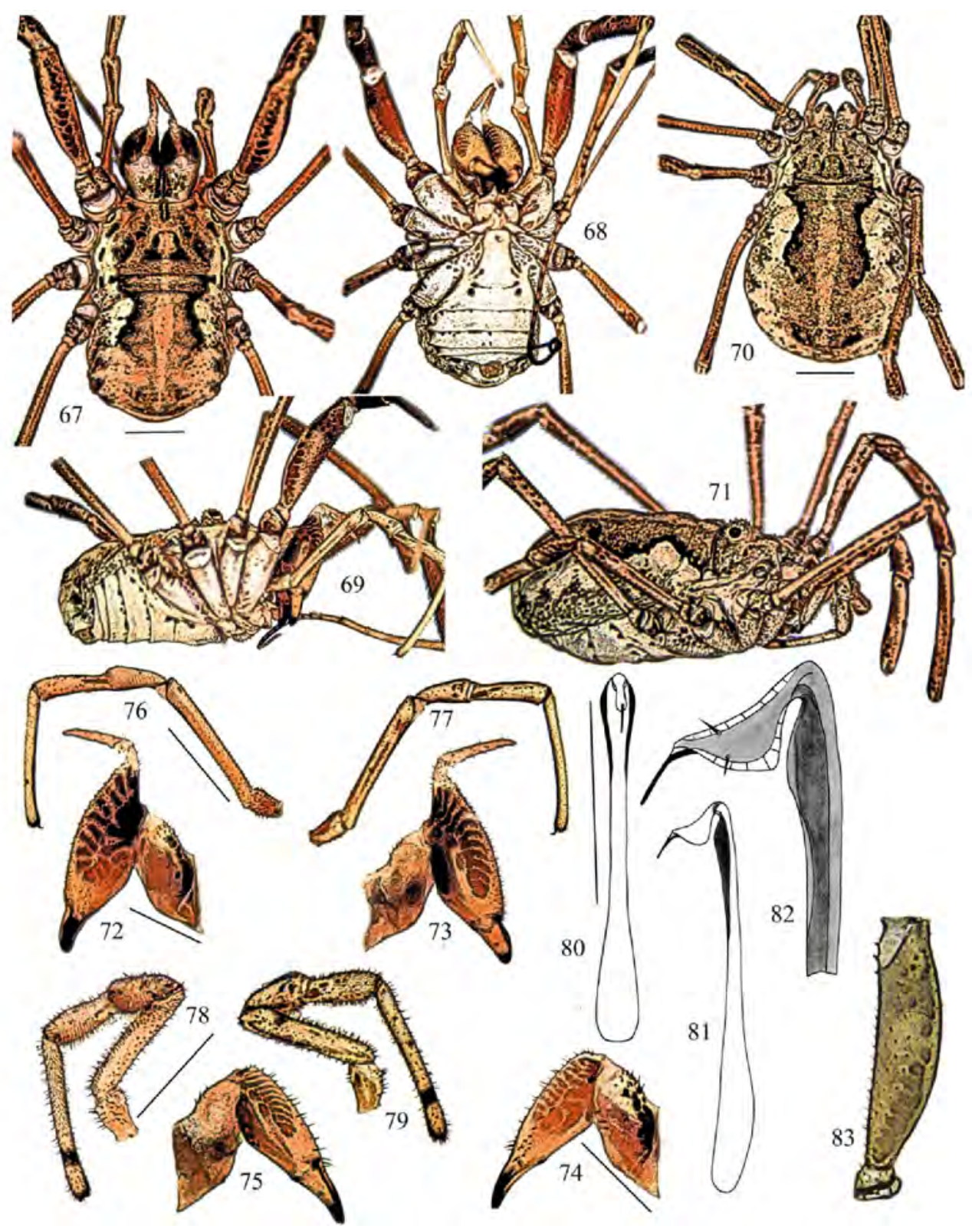

Figs 67-83. Phalangium bakuense Snegovaya, 2006: 67 - male body, dorsal view, 68 - male body, ventral view, 69 male body, ventrolateral view, 70 - female body, dorsal view, 71 - female body, anterolateral view, 72 - male chelicerae, lateral view, 73 - male chelicerae, lateral view, 74 - female chelicerae, lateral view, 75 - female chelicerae, lateral view, 76 - male pedipalp, lateral view, 77- male pedipalp, lateral view, 78 - female pedipalp, lateral view, 79 female pedipalp, lateral view, 80 - penis, dorsal view, 81 - penis, lateral view, 82 - glans, lateral view, 83 - femur I. Scale bars $=1 \mathrm{~mm}$ 


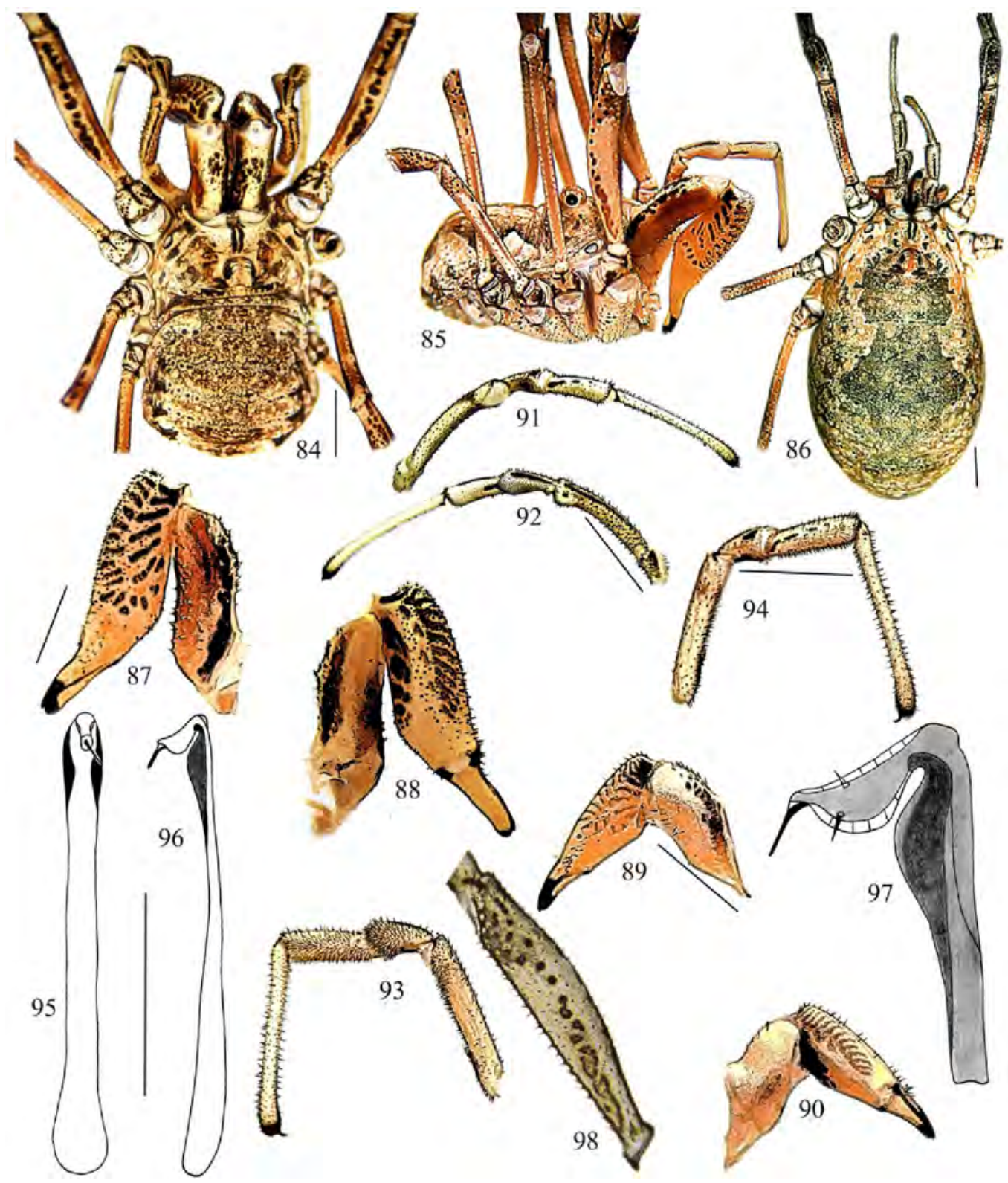

Figs 84-98. Phalangium armenicum Chemeris, 2012: 84 - male body, dorsal view, 85 - male body, lateral view, 86 female body, dorsal view, 87 - male chelicerae, lateral view, 88 - male chelicerae, lateral view, 89 - female chelicerae, lateral view, 90 - female chelicerae, lateral view, 91 - male pedipalp, lateral view, 92 - male pedipalp, lateral view, 93 - female pedipalp, lateral view, 94 - female pedipalp, lateral view, 95 - penis, dorsal view, 96 - penis, lateral view, 97 - glans, lateral view, 98 - femur I. Scale bars $=1 \mathrm{~mm}$. 

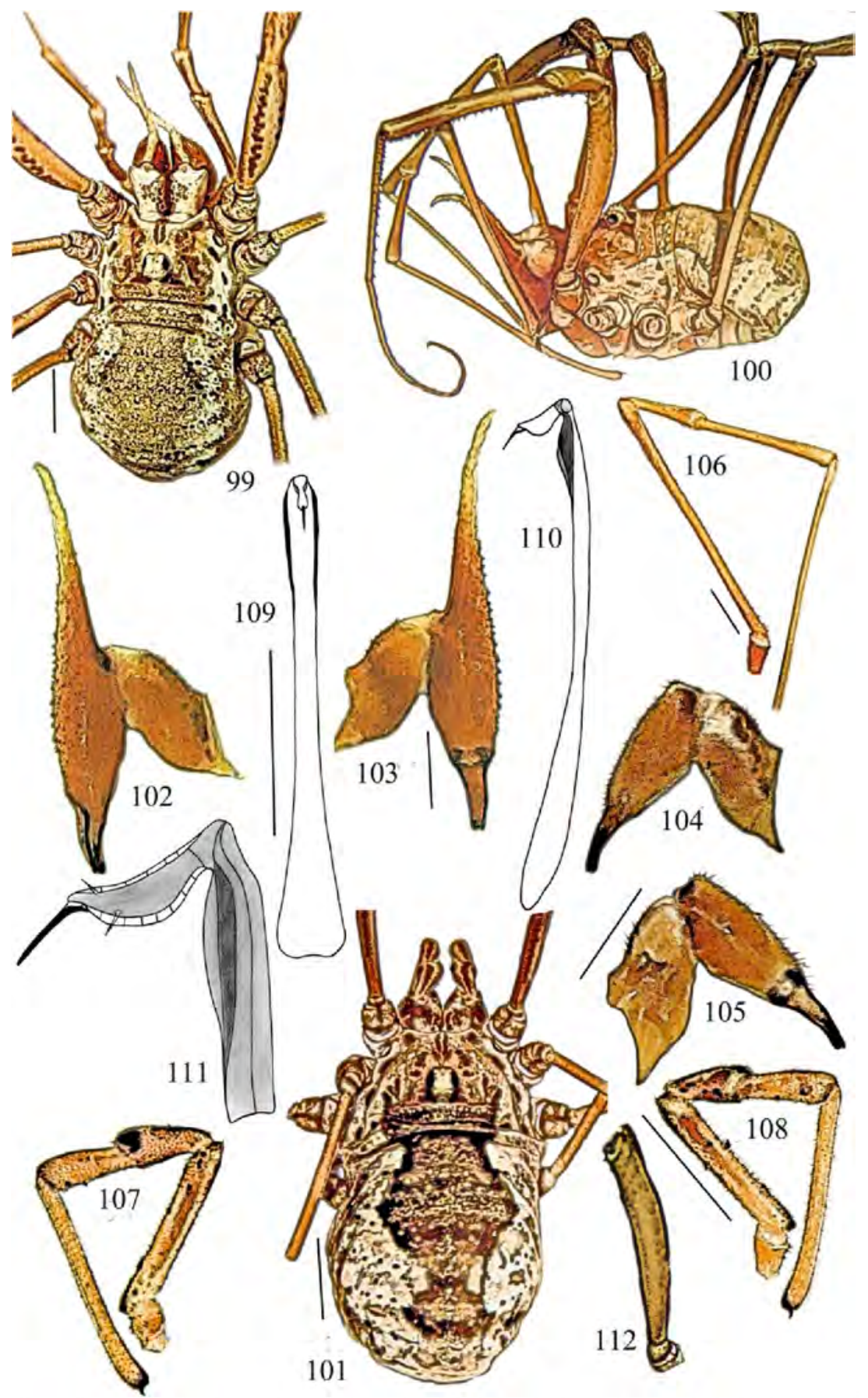

Figs. 99-112. Phalangium mcheidzeae sp. n.: 99 - male body, dorsal view, 100 - male body, lateral view, $101-$ female body, dorsal view, 102 - male chelicerae, lateral view, 103 - male chelicerae, lateral view, 104 - female chelicerae, lateral view, 105 - female chelicerae, lateral view, 106 - male pedipalp, lateral view, 107 - female pedipalp, lateral view, 108 - female pedipalp, lateral view, 109 - penis, dorsal view, 110 - penis, lateral view, 111 glans, lateral view, $112-$ femur I. Scale bars $=1 \mathrm{~mm}$. 


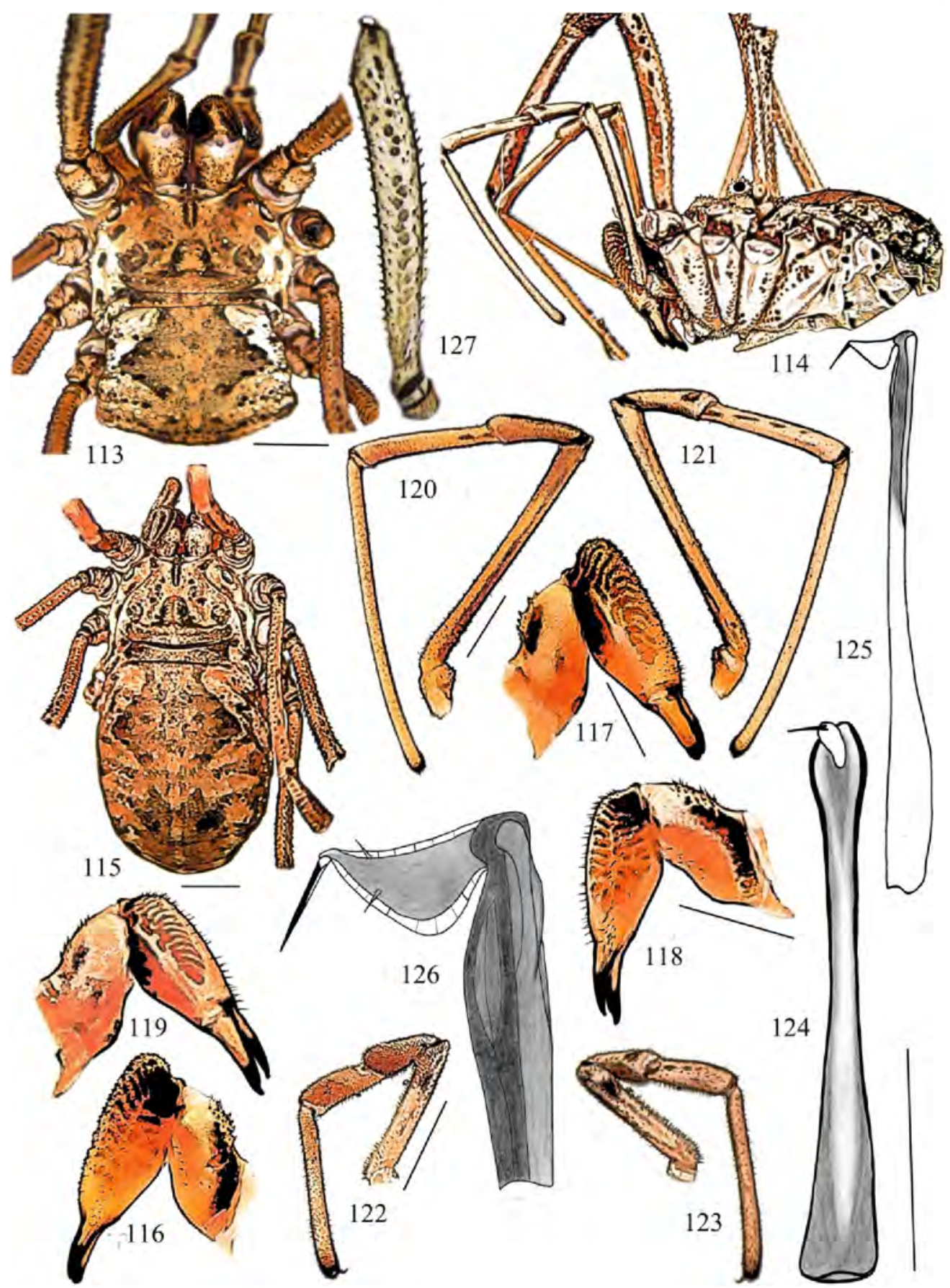

Figs. 113-127. Phalangium gorbunovi sp. n.: 113 - male body, dorsal view, 114 - male body, lateral view, 115 female body, dorsal view, 116 - male chelicerae, lateral view, 117- male chelicerae, lateral view, 118 - female chelicerae, lateral view, 119 - female chelicerae, lateral view, 120 - male pedipalp, lateral view, 121 - male pedipalp, lateral view, 122 - female pedipalp, lateral view, 123 - female pedipalp, lateral view, 124 - penis, dorsal view, 125 penis, lateral view, $126-$ glans, lateral view, $127-$ femur I. Scale bars $=1 \mathrm{~mm}$ 


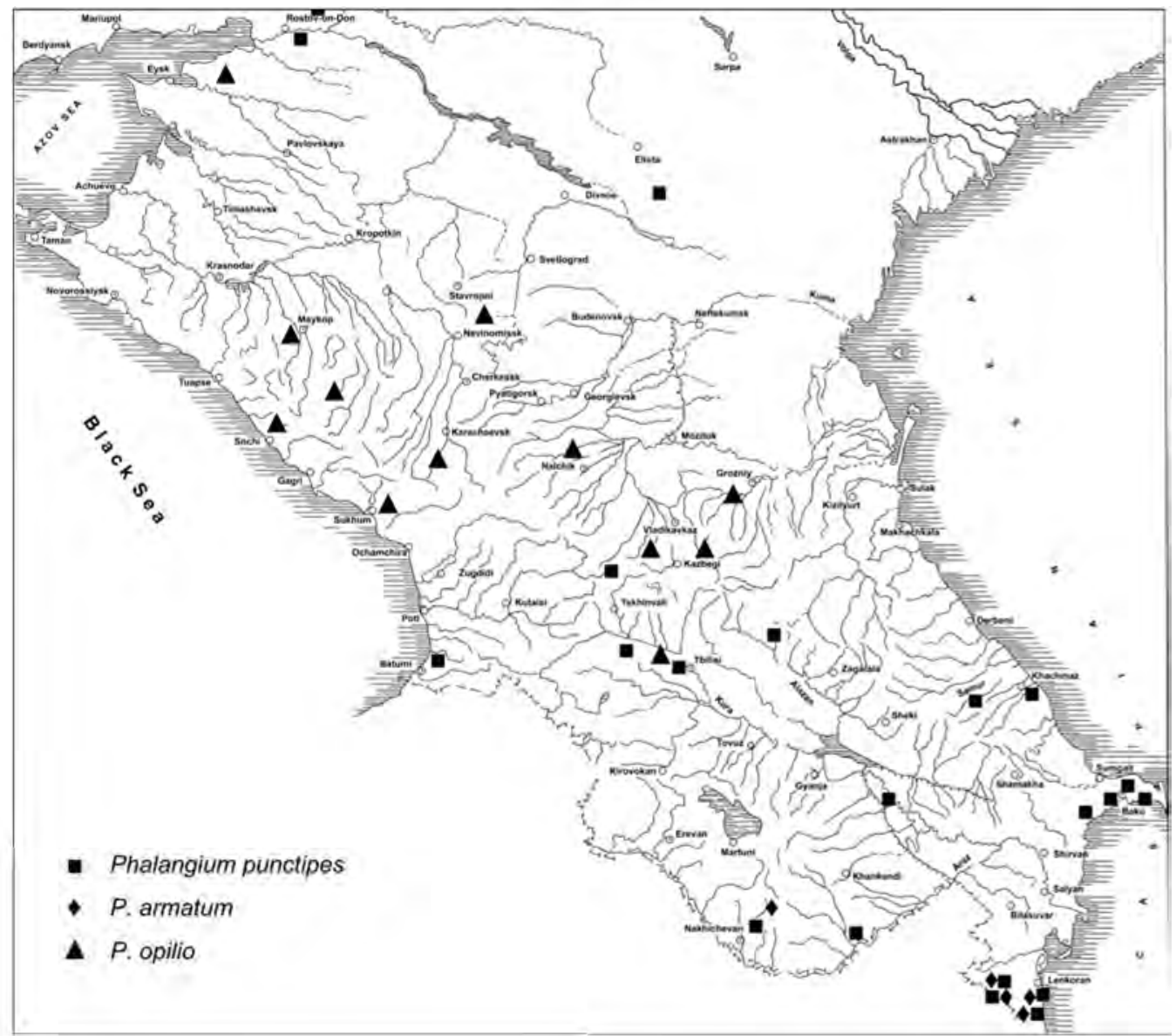

Fig. 128. Distributions of Phalangium opilio Linnaeus, 1758, P. punctipes (L. Koch. 1878) and P. armatum Snegovaya, 2005 in the Caucasus.

\section{IDENTIFICATION KEY TO MALES OF PHAL ANGIUM SPECIES FROM THE CAUCASUS.}

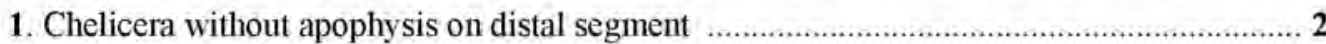

- Chelicera with apophysis 2

2. Cheliceral fingers strongly antero-ventrally bent P. armenicum

- Cheliceral fingers not bent or only slightly bent 3

3. Proximal leg segments with setae and small denticles ................................ P. punctipes

- Proximal leg segments with large denticles

4. Cheliceral apophysis rigid P. gorbunovi

- Cheliceral apophysis soft 5

5. Penis shaft strongly narrowed in the middle, with a conspicuous median keel P. opilio

- Penis shaft slightly narrowed in the middle, without keel 7

6. Body and legs with large denticles ... 6

- Body and legs with small denticles and setae P. armatum

7. Penis long $(>2.8 \mathrm{~mm})$, with very wide wing-shaped lateral keels P. mcheidzeae

- Penis short $(<2 \mathrm{~mm})$, with narrow wing-shaped lateral keels P. staregai P. bakuense 


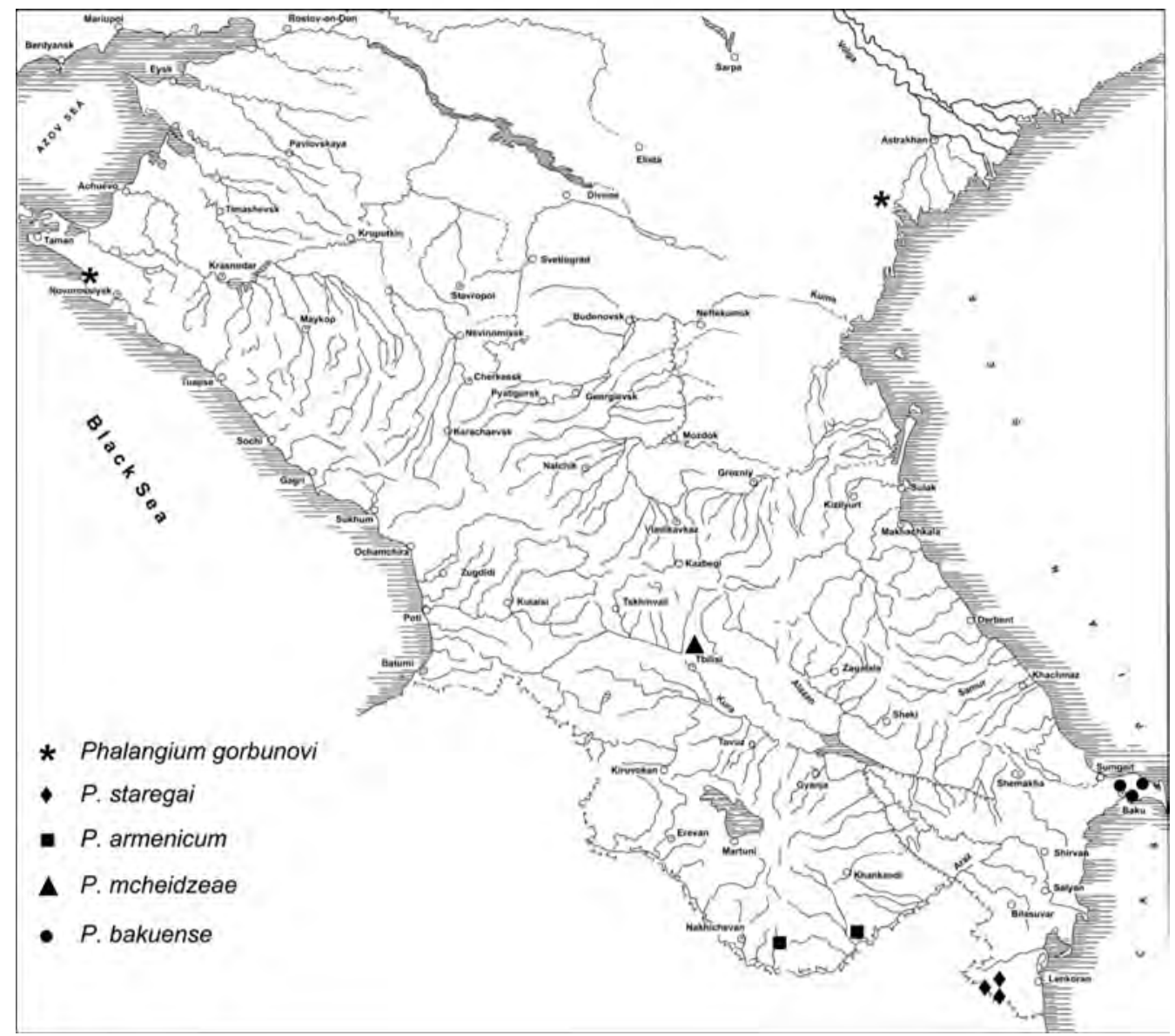

Fig. 129. Distributions of Phalangium starega Snegovaya, 2005, P. baknense Snegovaya, 2006, P. armenicum Chemeris, 2012, P. mcheidzeae sp.n. and P. gorbunovi sp.n. in the Caucasus.

\section{ACKNOWLEDGEMENTS}

I thank all the people who kindly provided specimens for the study - Drs Kh. Aliyev, E. Guseinov (Baku, Azerbaijan), Drs D. Kasatkin, M. Nabozhenko, A. Ponomarev (Rostov-naDonu, Russia), Dr Yu. Chumachenko (Adygeya, Maykop, Russia) and other colleagues. Further, I am much obliged to Dr V. Pkhakadze (Tbilisi, Georgia) for access to the material kept in the National Museum of Georgia, Tbilisi. I am grateful to Dr J. Cokendolpher (Lubbock, Texas, USA) for critical remarks and improving the English. I thank Dr J. Wytwer (Warszawa, Poland) and two anonymous reviewers for valuable critical comments.

\section{REFERENCES}

CHEMERIS A. N. 2012. Three new species of the genus Phalangium Limnaeus, 1758 (Arachnida: Opiliones: Phalangiidae) from Armenia and Uzbekistan. Arthropoda Selecta 21: 13-24.

CHEmeris A. N., Kovblyuk M. M. 2005. A contribution to the knowledge of the harvestman fauna of the Crimea (Arachnida: Opiliones). Arthropoda Selecta 14:305-328.

Chemeris A. N., Logunoy D. V. \& Tsurusaki N. 1998. A contribution to the knowledge of the harvestman fauna of Siberia (Arachnida: Opiliones). Arthropoda Selecta 7: $189-199$. 
CHEvrZov B. P. 1979. Kratkiy opredelitel' senokostsev (Opiliones) evropeykoy chasti SSSR. A brief key to the harvestmen (Opiliones) from the European part of the USSR. The fauna and ecology of Arachnida. Proceedings of the Zoological Institute 8: 4-27 [in Russian].

KOCH L. 1878. Kaukasische Arachnoiden. In: SCHNEDER O. (ed.), Naturwissenschaftliche Beiträge zur Kenntniss der Kaukasusländer. Part III. Dresden, pp. 36-71.

MARTENs J. 1978.Spinnentiere, Arachnida: Weberknechte, Opiliones. Die Tierwelt Deutschlands. Vol. 64. G. Fischer Verlag, Jena 1-464.

MARUSIK YU. M., OtTo S. 2008. 70 years of Arachnology in Georgia: Tamara S. Mkheidze 1915-2007. The Newsletter of the British Arachnological Society. 112: 6-8.

MCHEIDZE T. S. 1959. Masalebi mtibavebis (Opiliones) sakhebrivi medbenilobisa da bavrts'elebis mesnavlisatvis sakartvelomi / Materials to the study of species composition and distribution of harvestmen in Georgia. Tbilisis Universitetis sromebi [Trudy Tbilisskogo gosudarstvennogo Universiteta im Stalina; Proceedings of Tbilisi University], Tbilisi, 70, 109-117 [in Georgian with Russian summary].

MCHEIDZE T. S. 1962. Kharagaulis raionis obobisnairta shesnavlisatvis [A study of Arachnoidea from Kharagaul district]. Tbilisis Universitetis sromebi [Trudy Tbilisskogo Universiteta; Proceedings of Tbilisi University], Tbilisi, 72 ["1960"], 183-189 [in Georgian].

MCHEIDZE T. S. 1964. Mtibavebi (Opiliones)/Opilionidea. In: Sakartvelos echoveloa samgaro, 2. Pechsachsrianebi. [Tierwelt in Grusien, 2. Arthropoda]. Tbilisi, pp. 117-126 [in Georgian].

RoEwer W. 1911. Übersicht der Genera der Subfamilie der Phalangiini der Opiliones Palpatores nebst Beschreibung einiger neuer Gattungen und Arten. Archiv für Naturgeschichte, Berlin, Abt. A, Original-Arbeiten 77: 1-106.

RoEweR W. 1912. Revision der Opiliones Palpatores (= Opiliones Plagiostethi). II. Teil: Familie der Phalangiidae. (Subfamilien: Sclerosomini, Oligolophini, Phalangiini). Abhandlungen aus dem Gebiete der Naturwissenschaften, herausgegeben vom Naturwissenschaftlichen Verein in Hamburg 20 (1): 1-295.

ROEWER W. 1923. Die Weberknechte der Erde. Systematische Bearbeitung der bisher bekannten Opiliones. Gustav Fischer, Jena $1116 \mathrm{pp}$.

Rozwer W. 1956. Über Phalangiinae (Phalangiidae, Opiliones Palpatores). (Weitere Weberknechte XIX). Senckenbergiana Biologica, Frankfurt, 37 (3/4): 247-318.

ŠLHAVÝ V. 1956. Sekáči - Opilionidea. Fauna ČSR, No. 7. Nakladatelství Československé Akademie Věd, Praha, $1-272$ [in Czech]

SNEgOvayA N.YU. 1999. Contribution to the harvest spider (Arachnida, Opiliones) fauna of the Caucasus. Turkish Journal of Zoology 23: 453-459.

SNEGovayA N.YU. 2004. Preliminary notes on the harvestman fauna (Opiliones) of Azerbaijan. In: LoGUNOV D.V. \& PENNEY D. (eds). European Arachnology 2003: 307-318

SnEgovaya N.YU. 2005. Four new harvestman species from Azerbaijan (Arachnida: Opiliones: Phalangiidae). Arthropoda Selecta 14 (1): 19-32

SNEGOVAYA N. YU. 2006. On the harvestman fauna of Absheron-Gobustan zone (Azerbaijan), with a description of a new species (Opiliones). Acta Zoologica Bulgarica, Supplementum 1: 95-100.

SNEGOVAYA N. YU. 2010. Novvie dannie po senokoscu Phalangium punctipes (L. Koch, 1878) (Arachnida, Opiliones, Phalangiidae) iz Azerbaidjana. Proceeding of the Azerbaijan Society of Zoologists. 2: 497-501 [in Russian].

Snegovaya N.YU. \& StaręGa W. 2009. Taurolaena, a new genus of Phalangiidae (Opiliones). Revista Ibérica de Aracnologia, Zaragoza, 17: 37-44.

Snegovaya N. YU. \& StaręGA W. 2011. Harvestmen (Arachnida, Opiliones) from Talysh, with description of a new genus and other taxonomical changes. Fragmenta Faunistica 54(1): 47-58.

STARĘGA W. 1966. Beitrag zur Kenntnis der Weberknecht-Fauna (Opiliones) der Kaukasusländer. Annales Zoologici, Warsawa 23: 387-411.

StaRĘGA W. 1973. Beitrag zur Kenntnis der Weberknechte (Opiliones) des Nahen Ostens. Annales Zoologici, Warsawa 30: 129-153.

StaREGA W. 1976. Die Weberknechte (Opiliones, excl. Sironidae) Bulgariens. Annales Zoologici 33 (18): $287-433$.

STARĘGA W. 1978. Katalog der Weberknechte (Opiliones) der Sowjet-Union. Fragmenta Faunistica, Warsaw 23: $197-241$.

YigIT N., BAYRAM A., ÇORAK I. \& DANISMAN T. 2007. External morphology of the male harvestman Phalangium opilio (Arachnida: Opiliones). Annals of the Entomological Society of America, 100(4): 574-581.

\section{STRESZCZENIE}

\section{[Przegląd kosarzy z rodzaju Phalangium Linnaeus, 1758 (Phalangiidae: Opiliones) z Kaukazu wraz z opisem dwóch nowych gatunków]}

Praca stanowi przegląd kosarzy z Kaukazu należących do rodzaju Phalangium Linnaeus, 1758 w oparciu o zbiory Muzeum Narodowego Gruzji w Tbilisi oraz zbiory z kolekcji Instytutu Zoologii Narodowej Akademii Nauk Azerbejdżanu. Na Kaukazie stwierdzono 
obecność 8 gatunków z rodzaju Phalangium, w tym dwa nowe dla nauki: $P$. mcheidzeae z Gruzji oraz $P$. gorbunovi $\mathrm{z}$ Regionu Wolgogradzkiego, Republiki Kalmuckiej i Krasnodarskiego Kraju. Przegląd podaje nie tylko opisy morfologiczne nowych gatunków z tego obszaru ale zawiera również ilustracje do pozostalych sześciu gatunków wykazanych z Kaukazu, tj. $P$. opilio, $P$. punctipes, $P$. armatum, $P$. staregai, $P$. bakuense i $P$. armenicum. Praca wzbogacona jest o analizę porównawczą kaukaskich gatunków Phalangium, zawiera także klucz do ich identyfikacji. Dane o rozmieszczeniu poszczególnych gatunków przedstawiono $\mathrm{w}$ postaci map. 